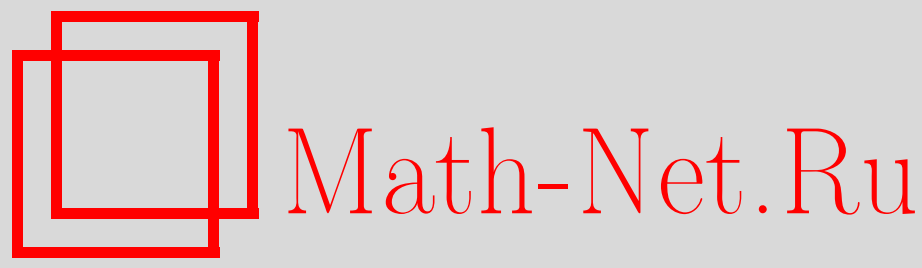

Н. М. Боголюбов, Четырехвершинная модель и случайные укладки, ТМ $\Phi, 2008$, том 155, номер 1, 25-38

DOI: https://doi.org/10.4213/tmf6190

Использование Общероссийского математического портала Math-Net.Ru подразумевает, что вы прочитали и согласны с пользовательским соглашением http: //www.mathnet.ru/rus/agreement

Параметры загрузки :

IP: 3.85 .5 .30

26 апреля 2023 г., $17: 42: 38$

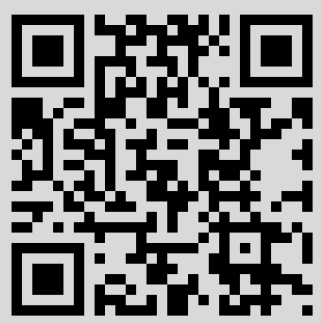




\title{
ЧЕТЫРЕХВЕРШИННАЯ МОДЕЛЬ И СЛУЧАЙНЫЕ УКЛАДКИ
}

\begin{abstract}
Рассмотрена точно решаемая четырехвершинная модель на квадратной решетке с различными граничными условиями. Применение алгебраического анзаца Бете позволяет вычислить статистическую сумму модели. Для фиксированных граничных условий установлена связь скалярного произведения векторов состояния модели с производящей функцией плоских разбиений, строгих по столбцам и строкам. Обсуждается соответствующая модель покрытий на периодической решетке.
\end{abstract}

Ключевые слова: интегрируемые модели, анзац Бете, плоские разбиения.

\section{1. ВВЕДЕНИЕ}

Связь интегрируемых моделей с комбинаторикой широко обсуждается в настоящее время, и важную роль в этих исследованиях играет шестивершинная модель. Эта модель интенсивно изучалась как для периодических, так и для фиксированных граничных условий [1]-[6]. Шестивершинная модель с граничными условиями типа доменной стенки имеет отношение к перечислению укладок "ацтекских алмазов" и к перечислению матриц чередующихся знаков [7]-[9].

Четырехвершинная модель является частным случаем шестивершинной модели, из которой исключены две вершины. Данная модель рассматривалась в работах [10], [11], где была выявлена ее связь с моделью случайных укладок на полубесконечном цилиндре. Модели укладок являются моделями классической дискретной статистической физики, суть их состоит в описании способов укладки определенных поверхностей плитками различной геометрической формы без зазоров и наложений. Укладки называются случайными лишь для того, чтобы отличить их от правильных квазипериодических покрытий.

В данной статье мы применяем квантовый метод обратной задачи (КМO3) к решению модели [12], [13]. Данный подход позволяет исследовать модель на решетке конечного размера с различными граничными условиями и вычислить статистические суммы вершинной модели и соответствующей ей модели покрытий. В частности, мы показываем, что статистическая сумма модели с фиксированными

${ }^{*}$ Санкт-Петербургское отделение Математического института им. В. А. Стеклова РАН, Санкт-Петербург, Россия. E-mail: bogoliub@pdmi.ras.ru 
граничными условиями имеет смысл производящей функции плоских разбиений, строгих по столбцам и строкам. Плоские разбиения эквивалентны как трехмерным диаграммам Юнга, помещенным в ящик конечного размера, так и покрытиям ромбами полурегулярного шестиугольника [14], [15]. В случае периодических граничных условий четырехвершинная модель эквивалентна модели покрытия тора единичными ромбами.

Связь интегрируемых моделей бозонного типа с теорией симметрических функций [14] и теорией плоских разбиений [16] рассматривалась в работах [17]-[20].

Статья организована следующим образом. В разделе 2 мы даем определение модели и излагаем подход КМОЗ к ее решению. В разделе 3 исследована модель на решетке с фиксированными граничными условиями и установлена связь этой модели с плоскими разбиениями. Статистическая сумма модели на периодической решетке вычислена в разделе 4 .

\section{2. ЧЕТЫРЕХВЕРШИННАЯ МОДЕЛЬ}

Четырехвершинная модель на квадратной решетке, являющаяся частным случаем шестивершинной модели, определяется четырьмя различными конфигурациями стрелок, направленными как в сторону каждого узла решетки, так и от него. Из шести возможных конфигураций две, с двумя горизонтальными стрелками, направленными направо, исключены. Статистический вес $\omega$ приписывается каждой допустимой конфигурации. Мы рассмотрим модель, в которой вершинные веса третьей и четвертой вершин равны (считая конфигурации на рис. 1 слева направо). Изображая стрелки, направленные вверх или направо жирными линиями, мы получим альтернативное описание вершин с помощью линий, проходящих сквозь узлы решетки. Так как грани решетки могут находиться лишь в двух состояниях (с жирной линией или без нее), то существует взаимно однозначное соответствие между допустимыми конфигурациями стрелок на решетке и графами линий - наборами решеточных путей.
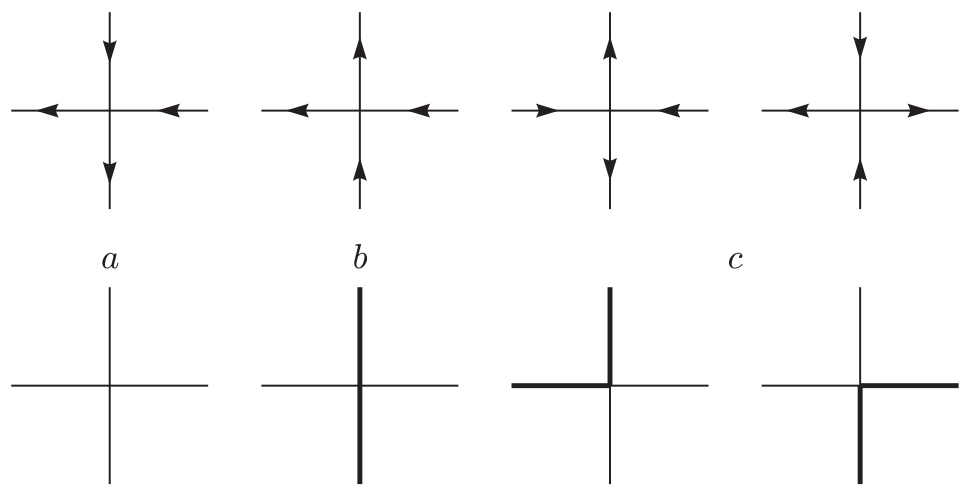

$c$

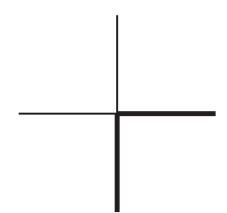

Рис. 1. Четыре допустимые вершины как конфигурации стрелок и линий. 
Для однородной модели вершинные веса $\omega$ не зависят от положения узла решетки, и статистическая сумма равна

$$
Z\left(\omega_{a}, \omega_{b}, \omega_{c}\right)=\sum \omega_{a}^{l^{a}} \omega_{b}^{l^{b}} \omega_{c}^{l^{c}},
$$

где суммирование ведется по всем допустимым конфигурациям стрелок на решетке, а $\omega_{a}, \omega_{b}$ и $\omega_{c}$ суть веса соответствующих вершин. Допустимые конфигурации зависят от наложенных граничных условий, которые определяются направлением стрелок на границе решетки. Число вершин типов $a, b, c$ в каждой конфигурации равно $l^{a}, l^{b}, l^{c}$ соответственно. Для неоднородной модели вершинные веса зависят от положения узла решетки.

Для того чтобы применить КМO3 для исследования данной модели, мы воспользуемся ее спиновым описанием. Каждому вертикальному ребру и каждому горизонтальному ребру решетки ставится в соответствие локальное пространство $\mathcal{C}^{2}$. Состояния со спином вверх и спином вниз образуют естественный базис в этом пространстве. Состояние со спином вверх на вертикальном ребре соответствует стрелке, направленной вверх, а состояние со спином вниз - стрелке, направленной вниз. Состояние со спином вверх на $i$-м горизонтальном ребре соответствует стрелке, направленной налево, $\left(\begin{array}{l}1 \\ 0\end{array}\right)_{i} \equiv|\leftarrow\rangle_{i}$, а состояние со спином вниз - стрелке, направленной направо, $\left(\begin{array}{l}0 \\ 1\end{array}\right)_{i} \equiv|\rightarrow\rangle_{i}$. Вспомогательным пространством мы будем называть пространство $\mathcal{V}=\left(\mathcal{C}^{2}\right)^{\otimes N}$, ассоциированное со всеми вертикальными ребрами решетки, а квантовым пространством - пространство $\mathcal{H}=\left(\mathcal{C}^{2}\right)^{\otimes N}$, ассоциированное со всеми горизонтальными ребрами решетки. В каждом узле решетки в пространстве $\mathcal{V} \otimes \mathcal{H}$ действует оператор. Этот оператор, действующий нетривиально только в локальном вспомогательном пространстве $\mathcal{C}^{2}$ и локальном квантовом пространстве $\mathcal{C}^{2}$, называется $L$-оператором.

$L$-оператор четырехвершинной модели имеет вид

$$
L(n \mid u)=-\frac{u}{2}\left(1+\sigma^{z}\right) e_{n}+\frac{u^{-1}}{2}\left(1-\sigma^{z}\right) e_{n}+\sigma^{+} \sigma_{n}^{-}+\sigma^{-} \sigma_{n}^{+},
$$

где параметр $u \in \mathcal{C}, \sigma^{z, \pm}$ - матрицы Паули, проектор $e=\left(\sigma^{z}+1\right) / 2$. Подстрочный индекс $n$ означает, что матрица действует нетривиально только в $n$-м квантовом пространстве: $s_{n}=I \otimes \cdots \otimes I \otimes s \otimes I \otimes \cdots \otimes I$.

Матричные элементы введенного $L$-оператора

$$
L(n \mid u)=\left(\begin{array}{ll}
L_{11}(n \mid u) & L_{12}(n \mid u) \\
L_{21}(n \mid u) & L_{22}(n \mid u)
\end{array}\right)=\left(\begin{array}{cc}
-u e_{n} & \sigma_{n}^{-} \\
\sigma_{n}^{+} & u^{-1} e_{n}
\end{array}\right)
$$

можно представить в виде точек с примыкающими стрелками (рис. 2). Матричный элемент $L_{11}(n \mid u)$ соответствует вершине 1 на рис. 2, где точкой обозначен оператор $-u e_{n}$, действующий в локальном квантовом пространстве. Единственный ненулевой элемент этого оператора ${ }_{n}\left\langle\leftarrow\left|-u e_{n}\right| \leftarrow\right\rangle_{n}$ определяет вершину $b$ на рис. 1 с весом $-u$. Матричный элемент $L_{22}(n \mid u)$ соответствует вершине 2 на рис. 2 с точкой, обозначающей оператор $u^{-1} e_{n}$. Этот оператор имеет ненулевой элемент ${ }_{n}\left\langle\leftarrow\left|u^{-1} e_{n}\right| \leftarrow\right\rangle_{n}$, который определяет вершину $a$ на рис. 1 с весом $u^{-1}$. Матричные элементы $L_{12}(n \mid u)=\sigma_{n}^{-}$и $L_{21}(n \mid u)=\sigma_{n}^{+}$соответствуют вершинам 3 и 4 на рис. 2 , ненулевые матричные элементы этих операторов ${ }_{n}\left\langle\rightarrow\left|\sigma_{n}^{-}\right| \leftarrow\right\rangle_{n}$ и ${ }_{n}\left\langle\leftarrow\left|\sigma_{n}^{+}\right| \rightarrow\right\rangle_{n}$ определяют вершины $c$ на рис. 1 с весом, равным единице. 


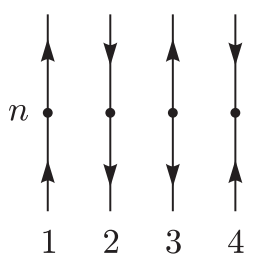

Рис. 2. Вершинное представление матричных элементов $L$-оператора.

Операторнозначная матрица (2) удовлетворяет сплетающему соотношению

$$
R(u, v)(L(n \mid u) \otimes L(n \mid v))=(L(n \mid v) \otimes L(n \mid u)) R(u, v),
$$

в котором $R(u, v)$ - это $(4 \times 4)$-матрица

$$
R(u, v)=\left(\begin{array}{cccc}
f(v, u) & 0 & 0 & 0 \\
0 & g(v, u) & 1 & 0 \\
0 & 0 & g(v, u) & 0 \\
0 & 0 & 0 & f(v, u)
\end{array}\right)
$$

где

$$
f(v, u)=\frac{u^{2}}{u^{2}-v^{2}}, \quad g(v, u)=\frac{u v}{u^{2}-v^{2}} .
$$

Вертикальная матрица монодромии определяется как произведение $L$-операторов:

$$
T(u)=L(M \mid u) L(M-1 \mid u) \ldots L(0 \mid u)=\left(\begin{array}{ll}
A(u) & B(u) \\
C(u) & D(u)
\end{array}\right) .
$$

Матричные элементы матрицы монодромии (7) выражаются через суммы по всем возможным конфигурациям стрелок на одномерной решетке с $M+1$ узлами (рис. 3 ). Например, оператору

$$
B(u)=\sum_{k_{M}, \ldots, k_{1}=1}^{2} L_{1 k_{M}}(M \mid u) L_{k_{M} k_{M-1}}(M-1 \mid u) \ldots L_{k_{1} 2}(0 \mid u)
$$

соответствуют граничные условия, при которых верхняя и нижняя стрелки направлены наружу (конфигурация $B$ на рис. 3). Оператору $C(u)$ соответствуют граничные условия, при которых верхняя и нижняя стрелки направлены внутрь (конфигурация $C$ на рис. 3). Операторам $A(u)$ и $D(u)$ соответствуют граничные условия, при которых верхняя и нижняя стрелки направлены в одном направлении (конфигурации $A$ и $D$ на рис. 3$)$.

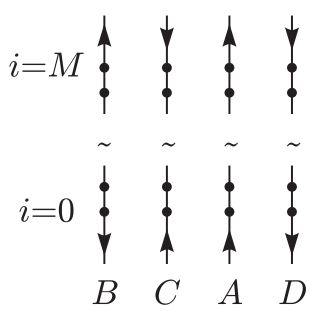

Рис. 3. Графическое представление матричных элементов матрицы монодромии. 
Коммутационные соотношения матричных элементов матрицы монодромии задаются той же $R$-матрицей (5):

$$
R(u, v)(T(u) \otimes T(v))=(T(v) \otimes T(u)) R(u, v),
$$

наиболее важными из которых являются следующие:

$$
\begin{aligned}
C(u) B(v) & =g(u, v)\{A(u) D(v)-A(v) D(u)\}, \\
A(u) B(v) & =f(u, v) B(v) A(u)+g(v, u) B(u) A(v), \\
D(u) B(v) & =f(v, u) B(v) D(u)+g(u, v) B(u) D(v), \\
{[B(u), B(v)] } & =[C(u), C(v)]=0 .
\end{aligned}
$$

Матрица перехода $\tau(u)$ определяется как след матрицы монодромии во вспомогательном пространстве:

$$
\tau(u)=\operatorname{tr} T(u)=A(u)+D(u) .
$$

Равенство (8) означает, что $[\tau(u), \tau(v)]=0$ при любых значениях параметров $u, v$.

$L$-оператор (2) удовлетворяет соотношению

$$
e^{\zeta \sigma_{n}^{z}} L(n \mid u) e^{-\zeta \sigma_{n}^{z}}=e^{-\zeta \sigma^{z} / 2} L(n \mid u) e^{\zeta \sigma^{z} / 2},
$$

где $\zeta$ - произвольный параметр. Из этого равенства и из определения матрицы монодромии (7) следует, что

$$
e^{\zeta S^{z}} T(u) e^{-\zeta S^{z}}=e^{-\zeta \sigma^{z}} T(u) e^{\zeta \sigma^{z}}
$$

где $S^{z}=\sum_{i=0}^{M} \sigma_{i}^{z} / 2$ является оператором $z$-компоненты полного спина. Равенства

$$
\begin{aligned}
& S^{z} B(u)=B(u)\left(S^{z}-1\right), \\
& S^{z} C(u)=C(u)\left(S^{z}+1\right)
\end{aligned}
$$

вытекают из соотношения (12) и означают, что оператор $B(u)$ понижает полный спин системы, а оператор $C(u)$ повышает его. Из равенства (12) следует, что

$$
\left[\tau(u), S^{z}\right]=0
$$

Производящим вектором квантового пространства $\mathcal{H}$ является состояние со всеми спинами, направленными вверх,

$$
|\Leftarrow\rangle=\bigotimes_{i=0}^{M}|\leftarrow\rangle_{i}=\bigotimes_{i=0}^{M}\left(\begin{array}{l}
1 \\
0
\end{array}\right)_{i} .
$$

Данный вектор уничтожается оператором $C(u)$,

$$
C(u)|\Leftarrow\rangle=0,
$$

и является собственным вектором операторов $A(u)$ и $D(u)$,

$$
A(u)|\Leftarrow\rangle=\alpha(u)|\Leftarrow\rangle, \quad D(u)|\Leftarrow\rangle=\delta(u)|\Leftarrow\rangle,
$$

с собственными значениями

$$
\alpha(u)=(-u)^{M+1}, \quad \delta(u)=\left(u^{-1}\right)^{M+1}
$$

соответственно. Полный спин производящего вектора равен $(M+1) / 2$ :

$$
S^{z}|\Leftarrow\rangle=\frac{1}{2}(M+1)|\Leftarrow\rangle .
$$


Одним из основных объектов дальнейшего рассмотрения будет состояние, полученное последовательным действием операторов $B(u)$ на состояние $|\Leftarrow\rangle$ :

$$
\left|\Psi_{n}\left(u_{1}, u_{2}, \ldots, u_{N}\right)\right\rangle=\prod_{i=1}^{N} B\left(u_{i}\right)|\Leftarrow\rangle .
$$

Из равенства (13) следует, что полный спин этого вектора равен

$$
S^{z} \prod_{i=1}^{N} B\left(u_{i}\right)|\Leftarrow\rangle=\frac{1}{2}(M+1-2 N) \prod_{i=1}^{N} B\left(u_{i}\right)|\Leftarrow\rangle .
$$

Сопряженный к (20) вектор определяется как

$$
\left\langle\Psi_{n}\left(u_{1}, u_{2}, \ldots, u_{N}\right)\right|=\langle\Leftarrow| \prod_{i=1}^{N} C\left(u_{i}\right) .
$$

Легко проверить, что $\langle\Leftarrow| B(u)=0$.

В координатном представлении вектор состояния имеет вид

$$
\left|\Psi_{N}\left(u_{1}, u_{2}, \ldots, u_{N}\right)\right\rangle=\prod_{k=1}^{N} B\left(u_{k}\right)|\Leftarrow\rangle=\sum \chi_{\mu}\left(u_{1}, u_{2}, \ldots, u_{N}\right)\left|m_{1}, \ldots, m_{N}\right\rangle,
$$

где через $\left|m_{1}, \ldots, m_{N}\right\rangle$ мы обозначили состояние с $N$ спинами, направленными вниз, в узлах $m_{1}, \ldots, m_{N}$, и с $M+1-N$ спинами, направленными вверх, в остальных узлах. Волновая функция $\chi_{\mu}\left(u_{1}, u_{2}, \ldots, u_{N}\right)$ удовлетворяет условию исключения. Она отлична от нуля только в том случае, когда числа $\mu=\left(m_{1}, m_{2}, \ldots, m_{N}\right)$ образуют строгое разбиение $M \geqslant m_{1}>m_{2}>\cdots>m_{N} \geqslant 0$, причем $m_{i} \geqslant m_{i+1}+2$. Прямым вычислением можно показать, что волновая функция выражается через определитель:

$$
\chi_{\mu}\left(u_{1}, u_{2}, \ldots, u_{N}\right)=(-1)^{\sum m_{k}}\left(u_{1} \ldots u_{N}\right)^{M-2(N-1)} \frac{\operatorname{det}\left(u_{j}^{-2\left(m_{k}+k-N\right)}\right)}{\prod_{1 \leqslant k<j \leqslant N}\left(u_{k}^{-2}-u_{j}^{-2}\right)} .
$$

Волновая функция, записанная через симметрическую функцию Шура

имеет вид

$$
S_{\lambda}\left(u_{1}, u_{2}, \ldots, u_{N}\right)=\frac{\operatorname{det}\left(u_{j}^{N-k+\lambda_{k}}\right)}{\prod_{1 \leqslant k<j \leqslant N}\left(u_{k}-u_{j}\right)},
$$

$$
\chi_{\mu}\left(u_{1}, u_{2}, \ldots, u_{N}\right)=(-1)^{\sum m_{k}}\left(u_{1} \ldots u_{N}\right)^{M-2(N-1)} S_{\mu-\delta}\left(u_{1}^{-2}, u_{2}^{-2}, \ldots, u_{N}^{-2}\right),
$$

где $\delta$ есть разбиение с элементами $\delta_{j}=2(N-j), j=1, \ldots, N$, а элементы разбиения $\lambda=\mu-\delta$ равны $\lambda_{j}=m_{j}-2(N-j)$ и удовлетворяют условию $M-2(N-1) \geqslant \lambda_{1} \geqslant$ $\lambda_{2} \geqslant \cdots \geqslant \lambda_{N} \geqslant 0$.

\section{3. ФИКСИРОВАННЫЕ ГРАНИЧНЫЕ УСЛОВИЯ}

Рассмотрим модель на квадратной решетке размера $2 N \times(M+1)$ со следующими граничными условиями: все стрелки на правой и левой границах направлены налево, верхние и нижние стрелки первых $N$ столбцов (считая слева) направлены внутрь, а верхние и нижние стрелки последних $N$ столбцов направлены наружу. Данные условия мы будем называть фиксированными граничными условиями. 
Для того чтобы перечислить все возможные конфигурации стрелок, значительно удобнее использовать описание модели в терминах линий и представлять допустимые конфигурации как наборы решеточных путей. Путь начинается в одной из $N$ нижних левых вершин, завершается в одной из $N$ верхних правых вершин и всегда идет на восток или север. Пути не соприкасаются, причем допустимы несколько последовательных шагов в вертикальном направлении и лишь один шаг в горизонтальном направлении. Если первые $N$ столбцов решетки имеют номера $-N, \ldots,-1$, а последние - номера $1, \ldots, N$, если нижняя строка имеет номер 0 , а верхняя номер $M$, тогда путь с номером $m$ идет из вершины $(-N+m-1 ; 0)$ к вершине $(m ; M), 1 \leqslant m \leqslant N$. Типичный набор путей изображен на рис. 4 . Длина пути равна $N+M$. Число вершин типа $c$ в допустимом пути равно $2 N$, так как в горизонтальном направлении разрешен лишь один последовательный шаг, число вершин типа $b$ равно $M-N+1$. Это означает, что число вершин типа $c$ и $b$ в наборе путей равно соответственно $l^{c}=2 N^{2}$ и $l^{b}=l^{a}=N(M-N+1)$.
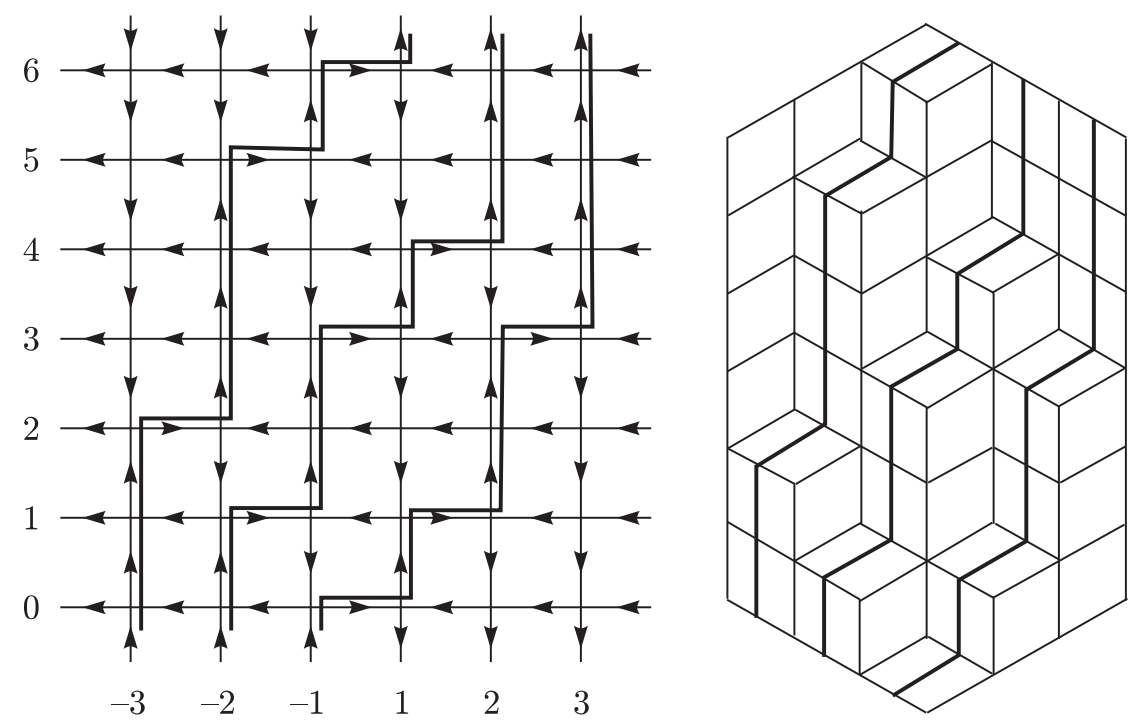

Рис. 4. Типичная конфигурация допустимых решеточных путей на решетке с фиксированными граничными условиями и соответствующее ей строгое плоское разбиение с градиентными линиями.

Статистическая сумма однородной модели с фиксированными граничными условиями равна

$$
Z\left(\omega_{a}, \omega_{b}, \omega_{c}\right)=\left(\omega_{a} \omega_{b}\right)^{N(M-N+1)} \omega_{c}^{2 N^{2}} S(N, M),
$$

где через $S(N, M)$ обозначено число всех допустимых конфигураций решеточных путей.

В случае неоднородной модели вершинные веса $\omega_{a}, \omega_{b}$ и $\omega_{c}$ зависят от положения узла решетки. Мы рассмотрим модель, в которой веса зависят только от номера столбца. При нашем способе нумерации столбцов статистическая сумма модели 
равна

$$
Z\left(\left\{\omega_{a}\right\},\left\{\omega_{b}\right\},\left\{\omega_{c}\right\}\right)=\sum \prod_{k=-1}^{-N}\left(\omega_{a}\right)_{k}^{l_{k}^{a}}\left(\omega_{b}\right)_{k}^{l_{k}^{b}}\left(\omega_{c}\right)_{k}^{l_{k}^{c}} \prod_{j=1}^{N}\left(\omega_{a}\right)_{j}^{l_{j}^{a}}\left(\omega_{b}\right)_{j}^{l_{j}^{b}}\left(\omega_{c}\right)_{j}^{l_{j}^{c}} .
$$

Рассмотрим скалярное произведение векторов состояния (20) и (22)

$$
W\left(u_{1}, \ldots, u_{N} ; v_{1}, \ldots, v_{N}\right)=\left\langle\Leftarrow\left|C\left(v_{1}\right) \ldots C\left(v_{N}\right) B\left(u_{1}\right) \ldots B\left(u_{N}\right)\right| \Leftarrow\right\rangle,
$$

где $\{u\}$ и $\{v\}$ - это наборы независимых параметров. Мы можем представить матричные элементы скалярного произведения (28) как двумерную квадратную решетку размера $2 N \times(M+1)$. Первые $N$ вертикальных линий решетки ассоциируются с операторами $C\left(v_{j}\right)$, а последние $N$ вертикальных линий - с операторами $B\left(u_{j}\right)$. Горизонтальные линии ассоциируются с локальными квантовыми пространствами, линия с номером $i$ соответствует квантовому пространству с индексом $i$. Из графического представления операторов $B(u)$ и $C(u)$ следует, что матричный элемент $(28)$ равен сумме всех допустимых конфигураций стрелок (или решеточных путей) на решетке с фиксированными граничными условиями (рис. 4):

$$
\begin{aligned}
W\left(u_{1}, \ldots, u_{N} ; v_{1}, \ldots, v_{N}\right) & =\sum \prod_{k=-1}^{-N}\left(-v_{-k}\right)^{l_{k}^{b}}\left(v_{-k}^{-1}\right)^{l_{k}^{a}} \prod_{j=1}^{N}\left(-u_{j}\right)^{l_{j}^{b}}\left(u_{j}^{-1}\right)^{l_{j}^{a}}= \\
& =(-1)^{M N} \sum \prod_{k=-1}^{-N} v_{-k}^{l_{k}^{b}-l_{k}^{a}} \prod_{j=1}^{N} u_{j}^{l_{j}^{b}-l_{j}^{a}} .
\end{aligned}
$$

Положим

$$
\begin{array}{llr}
\left(\omega_{a}\right)_{j}=v_{-j}^{-1}, & \left(\omega_{b}\right)_{j}=v_{-j}, & -N \leqslant j \leqslant-1 ; \\
\left(\omega_{a}\right)_{j}=u_{j}^{-1}, & \left(\omega_{b}\right)_{j}=u_{j}, & 1 \leqslant j \leqslant N \\
\left(\omega_{c}\right)_{j}=1 . &
\end{array}
$$

Тогда

$$
Z\left(\left\{\omega_{a}\right\},\left\{\omega_{b}\right\},\left\{\omega_{c}\right\}\right)=(-1)^{M N} W\left(u_{1}, \ldots, u_{N} ; v_{1}, \ldots, v_{N}\right) .
$$

Скалярное произведение (28) при произвольном значении параметров $u_{j}, v_{j}$ вычисляется с помощью перестановочных соотношений (9) и равно [13], [17]

$$
W\left(u_{1}, \ldots, u_{n} ; v_{1}, \ldots, v_{n}\right)=(-1)^{M N}\left\{\prod_{j>k} \frac{v_{j} v_{k}}{v_{k}^{2}-v_{j}^{2}} \prod_{l>m} \frac{u_{l} u_{m}}{u_{l}^{2}-u_{m}^{2}}\right\} \operatorname{det} H
$$

где матричные элементы $(N \times N)$-матрицы $H$ равны

$$
H_{j k}=\left\{\left(\frac{u_{k}}{v_{j}}\right)^{M-N+2}-\left(\frac{u_{k}}{v_{j}}\right)^{-M+N-2}\right\} \cdot \frac{1}{\frac{u_{k}}{v_{j}}-\left(\frac{u_{k}}{v_{j}}\right)^{-1}} .
$$

Формулы (32), (33) определяют решение неоднородной четырехвершинной модели и дают представление ее статистической суммы в виде определителя.

Статистическую сумму можно вычислить в явном виде, если выбрать вершинные веса в следующем виде:

$$
v_{j}=q^{-j / 2}, \quad u_{j}=q^{(j-1) / 2},
$$

где $q$ - больцмановский вес, $q=e^{-\nu}$ с $\nu>0$. В данной параметризации статистическая сумма модели (27) принимает вид

$$
Z(q)=\sum q^{\sum_{k=-1}^{-N} k\left(l_{k}^{a}-l_{k}^{b}\right) / 2+\sum_{j=1}^{N}(j-1)\left(l_{j}^{a}-l_{j}^{b}\right) / 2},
$$


где суммирование ведется по всем допустимым наборам решеточных путей. Подстановка параметризации (34) преобразует представление (32) в

$$
W_{q}(N, M)=(-1)^{N M+N(N-1) / 2}\left\{\prod_{j>k}\left(q^{(j-k) / 2}-q^{-(j-k) / 2}\right)^{-2}\right\} \operatorname{det} Q,
$$

где элементы матрицы $Q$ равны

$$
Q_{j k}=\frac{s^{(k+j-1) / 2}-s^{-(k+j-1) / 2}}{q^{(k+j-1) / 2}-q^{-(k+j-1) / 2}}, \quad s=q^{M-N+2} .
$$

Определитель матрицы $Q$ был вычислен в работе [7]:

$$
\begin{gathered}
\operatorname{det} Q=(-1)^{N(N-1) / 2}\left\{\prod_{j>k}\left(q^{(j-k) / 2}-q^{-(j-k) / 2}\right)^{2}\right\} \times \\
\times \prod_{1 \leqslant j, k \leqslant N} \frac{s^{1 / 2} q^{(j-k) / 2}-s^{-1 / 2} q^{-(j-k) / 2}}{q^{(k+j-1) / 2}-q^{-(k+j-1) / 2}} .
\end{gathered}
$$

Подстановка этого выражения в (36) дает

$$
\begin{aligned}
W_{q}(N, M) & =(-1)^{N M} q^{-N^{2}(M+2-2 N) / 2} \prod_{1 \leqslant j, k \leqslant N} \frac{1-q^{M-N+2+j-k}}{1-q^{k+j-1}}= \\
& =(-1)^{N M} q^{-N^{2}(M+2-2 N) / 2} \prod_{1 \leqslant j, k \leqslant N} \frac{1-q^{M+3-j-k}}{1-q^{k+j-1}},
\end{aligned}
$$

а статистическая сумма (35) равна

$$
Z(q)=q^{-N^{2}(M+2-2 N) / 2} \prod_{1 \leqslant j, k \leqslant N} \frac{1-q^{M+3-j-k}}{1-q^{k+j-1}} .
$$

По определению $Z(1)=S(N, M)$, и для однородной модели (26) получим следующий ответ:

$$
Z\left(\omega_{a}, \omega_{b}, \omega_{c}\right)=\left(\omega_{a} \omega_{b}\right)^{N(M-N+1)} \omega_{c}^{2 N^{2}} \prod_{1 \leqslant j, k \leqslant N} \frac{M-j-k+3}{j+k-1} .
$$

Для того чтобы связать четырехвершинную модель с моделью укладок, заметим, что каждый набор допустимых решеточных путей может быть представлен в виде матрицы $\pi_{i j}$ размера $N \times N$. Путь с номером $m$ соответствует $m$-му столбцу этой матрицы; матричный элемент $\pi_{j m}$ равен числу ячеек в последующем столбце $j$ решетки (начиная с правой) под $m$-м путем. Например, матрица

$$
\pi=\left(\begin{array}{lll}
6 & 4 & 3 \\
5 & 3 & 1 \\
2 & 1 & 0
\end{array}\right)
$$

соответствует набору путей на рис. 4 .

Набор $\pi_{i j}$ неотрицательных целых чисел, упорядоченных в порядке нестрогого убывания по индексам $i$ и $j(i, j=1,2, \ldots)$, называется плоским разбиением $\pi$ [14]. Числа $\pi_{i j}$ называются частями плоского разбиения. У плоского разбиения есть трехмерная диаграмма (диаграмма Юнга), представляющая собой множество столбцов, составленных из единичных кубов. Число $|\pi|=\sum \pi_{i j}$ называется ее объемом или весом плоского разбиения. Высота столбца диаграммы с координатами $(i, j)$ равна

2 Теоретическая и математическая физика, т. 155, № 1, 2008 г. 


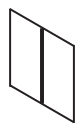

1

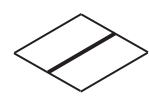

2

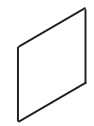

3

Рис. 5. Три типа единичных ромбов.

части плоского разбиения $\pi_{i j}$. Если $i \leqslant r, j \leqslant s$ и $\pi_{i j} \leqslant t$ для всех частей плоского разбиения, то такое плоское разбиение называется плоским разбиением, заключенным внутри ящика со сторонами $r, s, t$. Если при этом $\pi_{i j}>\pi_{i+1, j}$, т.е. части плоского разбиения убывают вдоль каждого столбца, то такое $\pi$ называется строгим по столбцам плоским разбиением. Плоское разбиение $\pi$ с частями, убывающими как по столбцам, так и по строкам $\left(\pi_{i j}>\pi_{i+1, j}\right.$ и $\left.\pi_{i j}>\pi_{i, j+1}\right)$, будем называть строгим разбиением. Если $s=r$, то элемент $\pi_{11}$ строгого разбиения $\pi_{i j}$ удовлетворяет условию $\pi_{11} \geqslant 2 r-2$. Произвольное плоское разбиение $\pi_{i j}$, заключенное в ящик со сторонами $r, r, t$, может быть преобразовано в строгое плоское разбиение в ящике со сторонами $r, r,(t+2 r-2)$, если добавить к нему разбиение, определяемое $(r \times r)$-матрицей

$$
\pi=\left(\begin{array}{cccc}
2 r-2 & 2 r-3 & \ldots & r-1 \\
2 r-3 & 2 r-4 & \ldots & r-2 \\
\vdots & \vdots & \ddots & \vdots \\
r-1 & r-2 & \ldots & 0
\end{array}\right)
$$

которая соответствует минимальному строгому разбиению.

Плоское разбиение в ящике со сторонами $r, s, t$ эквивалентно укладке полурегулярного шестиугольника единичными ромбами с углами $\pi / 3$ и $2 \pi / 3$ (рис. 5 ).

В рассматриваемом случае допустимые укладки представляют собой проекцию строгих трехмерных диаграмм Юнга с градиентными линиями. Трехмерная диаграмма Юнга, которая соответствует плоскому разбиению (41), изображена на рис. 4.

Статистическая сумма трехмерных диаграмм Юнга, заключенных в ящик, равна

$$
Z_{\mathrm{YD}}(q)=\sum q^{|\pi|}
$$

где $q$ - это больцмановский вес единичного куба, а суммирование ведется по всем диаграммам. Объем трехмерной строгой диаграммы Юнга в ящике со сторонами $N, N, M$ вычисляется по соответствующему ей набору решеточных путей [17]:

$$
|\pi|=\frac{N^{2} M}{2}+\sum_{k=-1}^{-N} \frac{k}{2}\left(l_{k}^{a}-l_{k}^{b}\right)+\sum_{j=1}^{N} \frac{j-1}{2}\left(l_{j}^{a}-l_{j}^{b}\right) .
$$

Подставляя это выражение в (35), мы получим следующий ответ для статистической суммы строгих трехмерных диаграмм Юнга в ящике со сторонами $N, N, M$ :

$$
Z_{\mathrm{YD}}(q)=q^{N^{2} M / 2} Z(q)=q^{N^{2}(N-1)} \prod_{1 \leqslant j, k \leqslant N} \frac{1-q^{M+3-j-k}}{1-q^{k+j-1}} .
$$

Эту статистическую сумму можно рассматривать как производящую функцию строгих плоских разбиений. Число соответствующих укладок шестиугольника единичными ромбами равно $Z_{\mathrm{YD}}(1)$ и совпадает со статистической суммой однородной мо- 
дели с единичными вершинными весами:

$$
Z_{\mathrm{YD}}(1)=Z(1,1,1)=\prod_{1 \leqslant j, k \leqslant N} \frac{M-j-k+3}{j+k-1} .
$$

\section{4. ПЕРИОДИЧЕСКИЕ ГРАНИЧНЫЕ УСЛОВИЯ}

Рассмотрим теперь модель на решетке с периодическими граничными условиями. Это означает, что на решетке с $M+1$ горизонтальными и $L$ вертикальными линиями граничные стрелки на любой линии направлены в одну сторону (рис. 6).
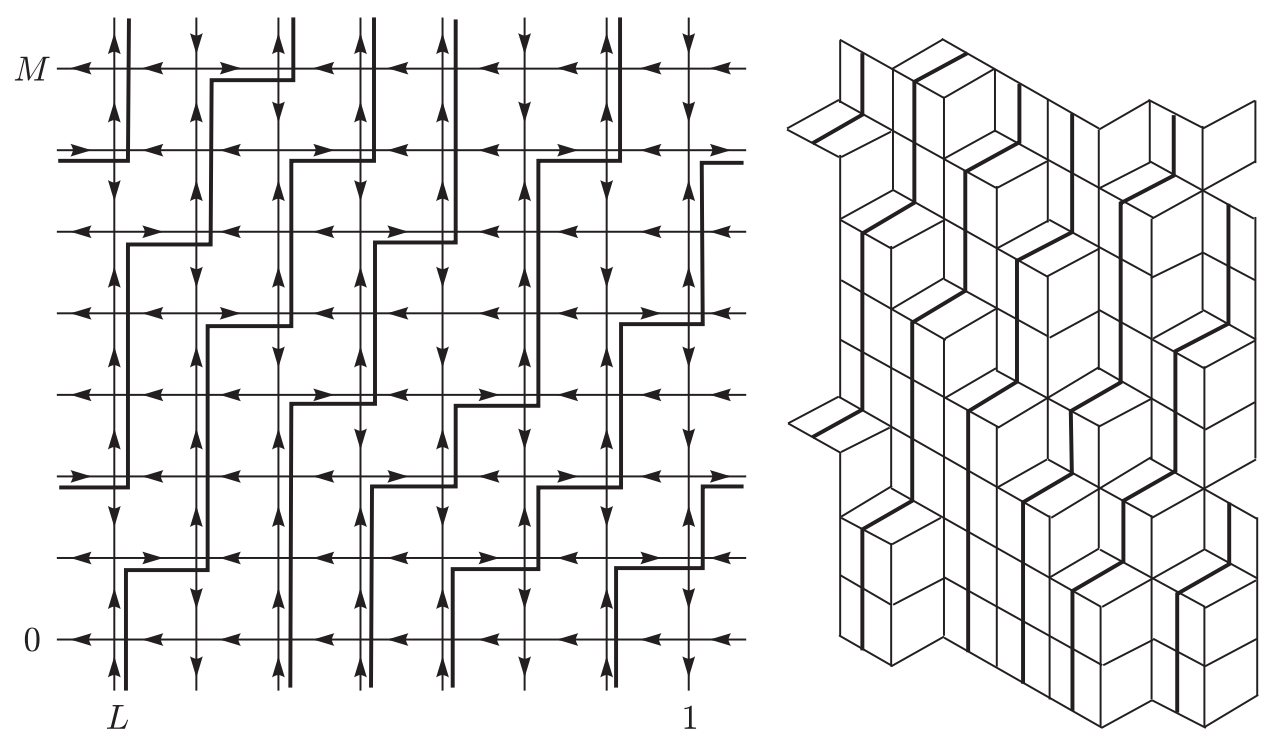

Рис. 6. Типичная конфигурация решеточных путей на решетке с периодическими граничными условиями и соответствующая ей укладка единичных ромбов.

Мы ограничимся случаем четных $M+1$ и $L$. В четырехвершинной модели число стрелок, направленных направо или налево в последующих столбцах решетки, сохраняется. Это означает, что число вершин типа $c$ на каждой вертикальной линии сохраняется и равно $l_{c}=2 n$, где $n$ - число стрелок, направленных направо в примыкающем столбце.

Статистическая сумма (1) однородной модели на периодической решетке размера $(M+1) \times L$ с фиксированным числом стрелок, направленных направо, и вершинными весами $\omega_{a}=e^{-\nu}, \omega_{b}=e^{\nu}, \omega_{c}=1$ равна

$$
Z_{n}(\mu)=\sum e^{\nu\left(l_{b}-l_{a}\right)}
$$

где суммирование ведется по всем допустимым конфигурациям стрелок на решетке с периодическими граничными условиями или по соответствующим наборам решеточных путей на рис. 6.

Рассмотрим след матрицы перехода в квантовом пространстве (10):

$$
\operatorname{tr} \tau^{L}(u)=\operatorname{tr}(A(u)+D(u))^{L}=\sum\left\langle m_{1}, \ldots, m_{n}\left|(A(u)+D(u))^{L}\right| m_{1}, \ldots, m_{n}\right\rangle .
$$


Здесь через $\left|m_{1}, \ldots, m_{n}\right\rangle$ обозначено состояние с $n$ спинами, направленными вниз в узлах $m_{1}, \ldots, m_{n}$, и с $M+1-n$ спинами, направленными вверх в остальных узлах, а суммирование ведется по полному набору состояний $\left|m_{1}, \ldots, m_{n}\right\rangle$ в квантовом пространстве $\mathcal{H}$. Сохранение числа $n$ есть следствие коммутативности (14) оператора третьей компоненты полного спина и матрицы перехода. Так как $\tau^{L}(u)$ является производящей функцией различных комбинаций операторов $A$ и $D$, то ее след (45) представляет собой сумму по всем допустимым конфигурациям вершин $a$, $b, c$ с весами $\omega_{a}=\delta(u), \omega_{b}=\alpha(u), \omega_{c}=1$ (18) на периодической решетке размера $(M+1) \times L:$

$$
\operatorname{tr} \tau^{L}(u)=\sum(-u)^{l^{b}}\left(u^{-1}\right)^{l^{a}} .
$$

Сравнивая это выражение с (44), мы получаем следующее представление для статистической суммы:

$$
Z_{n}(\nu)=e^{-i \pi(M+1-2 n) L / 2} \operatorname{tr} \tau^{L}\left(-i e^{\nu}\right),
$$

где мы положили $u=-i e^{\nu}$.

Для того чтобы вычислить это выражение, необходимо решить задачу на собственные значения для матрицы перехода $\tau(u)$ :

$$
\begin{gathered}
\tau(u)\left|\Psi_{n}\left(v_{1}, \ldots, v_{n}\right)\right\rangle=\Theta_{n}\left(u ; v_{1}, \ldots, v_{n}\right)\left|\Psi_{n}\left(v_{1}, \ldots, v_{n}\right)\right\rangle, \\
S^{z}\left|\Psi_{n}\left(v_{1}, \ldots, v_{n}\right)\right\rangle=\frac{1}{2}(M+1-2 n)\left|\Psi_{n}\left(v_{1}, \ldots, v_{n}\right)\right\rangle .
\end{gathered}
$$

Вектор (20) является собственным вектором матрицы перехода, если параметры $v_{j}$ удовлетворяют уравнениям Бете

$$
\begin{gathered}
\left(v_{k}^{2}\right)^{M+1-n}=(-1)^{n-1} V^{-2}, \quad k=1, \ldots, n, \\
V^{-2}=\prod_{j=1}^{n} v_{j}^{-2} .
\end{gathered}
$$

Положив $v_{k}^{2}=e^{i p_{k}}$, можно представить решения уравнений Бете (49) в виде

$$
p_{k}=\frac{2 \pi I_{k}-P}{M+1-n}, \quad-\pi<p_{k} \leqslant \pi,
$$

где $I_{n}$ - это целые или полуцелые числа в зависимости от того, является ли $n$ соответственно нечетным или четным числом, полный импульс $P=\sum_{j=1}^{n} p_{j}$. Классификацию решений уравнений (50) можно найти в работах [21], [22].

Собственное значение матрицы перехода равно

$$
\Theta_{n}(u ;\{v\})=e^{i \pi(M+1-2 n) / 2}\left\{u^{M+1} V^{-2}+(-1)^{n} u^{-(M+1-2 n)}\right\} \prod_{j=1}^{n} \frac{1}{u^{2}-v_{j}^{2}} .
$$

Статистическая сумма (44) четырехвершинной модели на периодической решетке задается равенством

$$
Z_{n}(\nu)=e^{-i \pi(M+1-2 n) L / 2} \sum_{\{v\}} \Theta_{n}^{L}\left(-i e^{\nu} ;\{v\}\right),
$$

где суммирование ведется по всем наборам решений уравнений Бете. Простейшее решение соответствует случаю $n=0$. При этом статистическая сумма равна $Z_{0}(\nu)=$ $\{2 \operatorname{ch}(M+1) \nu\}^{L}$ и $Z_{0}(0)=2^{L}$. Другой предельный случай соответствует $2 n=$ $M+1$. В этом случае существуют лишь два набора решений. Первый набор задается 
решениями (50) с $I_{k}=k-(n+1) / 2, k=1, \ldots, n$, и $P=0$. Второй набор получается при $I_{k}=k-(n+3) / 2, k=1, \ldots, n$, и $P=-\pi$. Применение равенства

$$
\prod_{k=1}^{n}\left(a-b e^{i 2 \pi k / n}\right)=a^{n}-b^{n}
$$

дает $Z_{(M+1) / 2}(\nu)=2$.

Вектором $\left|\Psi_{n}\left(p_{1}, \ldots, p_{n}\right)\right\rangle=\prod_{k=1}^{n} B\left(e^{i p_{k}}\right)|\Leftarrow\rangle$, сопряженным к собственному вектору (48), где $p_{k}$ - решения (50) уравнений Бете (49), является вектор

$$
\left\langle\Psi_{n}\left(p_{1}, \ldots, p_{n}\right)\right|=\langle\Leftarrow| \prod_{k=1}^{n} B^{\dagger}\left(e^{i p_{k}}\right) .
$$

Операторы $B\left(e^{i p}\right)$ и $C\left(e^{i p}\right)$ находятся в инволюции: $B^{\dagger}\left(e^{i p}\right)=(-1)^{M} C\left(e^{i p}\right)$.

Норма собственных векторов вычисляется с помощью представления (32). Когда наборы параметров совпадают, $\{u\}=\{v\}$, диагональные элементы матрицы $H$ равны

$$
H_{j j}=M-n+2 .
$$

Если $\{u\}=\{v\}$ являются решениями уравнений Бете (49), то недиагональные элементы матрицы $H$ равны единице: $H_{j k}=1$. Определитель легко вычисляется,

$$
\operatorname{det} H=(M+1)(M-n+1)^{n-1},
$$

и норма любого собственного вектора равна

$$
\begin{aligned}
\mathcal{N}^{2}\left(p_{1}, \ldots, p_{n}\right) & =\left\langle\Psi_{n}\left(p_{1}, \ldots, p_{n}\right) \mid \Psi_{n}\left(p_{1}, \ldots, p_{n}\right)\right\rangle= \\
& =(M+1)(M-n+1)^{n-1} \prod_{j \neq k} \frac{e^{i P}}{e^{i p_{j}}-e^{i p_{k}}},
\end{aligned}
$$

где $p_{j}$ - решения уравнений (50). Собственные векторы (48) образуют полную ортогональную систему [22].

Рассмотрим цилиндр с длиной окружности, равной $M+1$, и высотой $L$. Вероятность того, что решеточные пути, берущие начало в узлах $\tilde{\mu}=\left(\widetilde{m}_{1}, \ldots, \widetilde{m}_{n}\right)$, $\widetilde{m}_{i} \geqslant \widetilde{m}_{i+1}+2$, покинут цилиндр в узлах $\mu=\left(m_{1}, \ldots, m_{n}\right), m_{i} \geqslant m_{i+1}+2$, определяется следующим равенством:

$$
\begin{aligned}
& \left\langle m_{1}, \ldots, m_{n}\left|\tau^{L}\left(-i e^{\nu}\right)\right| \widetilde{m}_{1}, \ldots, \widetilde{m}_{n}\right\rangle= \\
& =\mathcal{N}^{-2} \sum_{\{p\}}\left\langle m_{1}, \ldots, m_{n} \mid \Psi_{n}\left(p_{1}, \ldots, p_{n}\right)\right\rangle \Theta_{n}^{L}\left(-i e^{\nu} ;\left\{e^{i p}\right\}\right) \times \\
& \quad \times\left\langle\Psi_{n}\left(p_{1}, \ldots, p_{n}\right) \mid \widetilde{m}_{1}, \ldots, \widetilde{m}_{n}\right\rangle= \\
& =\mathcal{N}^{-2} \sum_{\{p\}} S_{\mu-\delta}\left(e^{i p_{1}}, e^{i p_{2}}, \ldots, e^{i p_{n}}\right) S_{\tilde{\mu}-\delta}\left(e^{-i p_{1}}, e^{-i p_{2}}, \ldots, e^{-i p_{n}}\right) \Theta_{n}^{L}\left(-i e^{\nu} ;\left\{e^{i p}\right\}\right),
\end{aligned}
$$

где суммирование ведется по всем решениям уравнений Бете; в данной формуле мы воспользовались представлением (25).

Каждой конфигурации решеточных путей на торе можно сопоставить укладку тора единичными ромбами по следующему правилу: ромбу 1 на рис. 5 соответствует вертикальное ребро, по которому проходит путь, ромбу 2 - аналогичное горизонтальное ребро, а ромбу 3 - свободные вертикальные ребра. Число укладок, которые определяются четырехвершинной моделью, дается равенством (52) при $\nu=0$. 


\section{5. ЗАКЛЮЧЕНИЕ}

Отметим, что, хотя рассмотренная четырехвершинная модель и является частным случаем шестивершинной, детерминантные представления для статистической суммы неоднородной модели на решетке с фиксированными граничными условиями нельзя получить простым предельным переходом, так как подобный ответ для шестивершинной модели неизвестен. То же можно сказать и про полученное представление среднего (53) через симметрические функции.

Квантовым гамильтонианом, коммутирующим с матрицей перехода четырехвершинной модели, является гамильтониан, получающийся в пределе бесконечной анизотропии из гамильтониана XXZ-цепочки Гейзенберга [21], [22]. Описанный в статье подход позволяет получать детерминантные представления для различных корреляционных функций соответствующей квантовой модели.

Благодарности. Данная работа частично поддержана CRDF (грант № RUMI2622-ST-04) и РФФИ (грант № 07-01-00358).

\section{Список литературы}

[1] Р. Бакстер, Точно решаемые модели в статистической механике, Мир, М., 1985.

[2] V.E. Korepin, Comm. Math. Phys., 86:3 (1982), 391-418.

[3] V. Korepin, P. Zinn-Justin, J. Phys. A, 33:40 (2000), 7053-7066.

[4] N. M. Bogoliubov, A. G. Pronko, M. B. Zvonarev, J. Phys. A, 35:27 (2002), 5525-5541.

[5] D. Allison, N. Reshetikhin, Ann. Inst. Fourier (Grenoble), 55:6 (2005), 1847-1869.

[6] O. Syljuåsen, M. Zvonarev, Phys. Rev. E, 70:1 (2004), 016118.

[7] G. Kuperberg, Int. Math. Res. Not., 1996:3 (1996), 139-150.

[8] F. Colomo, A. G. Pronko, J. Stat. Mech., 2005, № 1, 01005.

[9] P. L. Ferrari, H. Spohn, J. Phys. A, 39:33 (2006), 10297-10306; arXiv: cond-mat/0605406.

[10] W. Li, H. Park, M. Widom, J. Phys. A, 23:11 (1990), L573-L580.

[11] W. Li, H. Park, J. Phys. A, 24 (1991), 257-264.

[12] L. D. Faddeev, 1 (1980), 107-155.

[13] V.E. Korepin, N. M. Bogoliubov, A. G. Izergin, Quantum Inverse Scattering Method and Correlation Functions, Cambridge University Press, Cambridge, 1993.

[14] И. Макдональд, Симметрические функиии и многочлены Холла, Мир, М., 1985.

[15] D. M. Bressoud, Proofs and Confirmations: the Story of the Alternating Sign Matrix Conjecture, Cambridge University Press, Cambridge, 1999.

[16] А. М. Вершик, Функи. анализ и его прил., 30:2 (1996), 19-39.

[17] N. M. Bogoliubov, J. Phys. A, 38:43 (2005), 9415-9430.

[18] Н. В. Цилевич, Функи. анализ и его прил., 40:3 (2006), 53-65.

[19] K. Shigechi, M. Uchiyama, J. Phys. A, 38:48 (2005), 10287-10306.

[20] Н. М. Боголюбов, ТМФ, 150:2 (2007), 193-203.

[21] М. Годен, Волновая функиия Бете, Мир, М., 1987.

[22] Н. И. Абаренкова, А. Г. Пронько, ТМФ, 131:2 (2002), 288-303. 